\title{
Transport des lipides chez les ruminants *
}

\author{
D Bauchart \\ INRA, laboratoire Croissance et métabolismes des herbivores, Theix, \\ 63122 Saint-Genès-Champanelle, France
}

Les lipides d'origine alimentaire ou endogène sont transportés dans le sang sous forme de complexes pseudomicellaires, les lipoprotéines, associant des lipides hydrophobes (triglycérides, TG, esters de cholestérol, EC) enrobés par une enveloppe hydrophile constituée d'apoprotéines, de phospholipides $(P L)$ et de cholestérol libre.

Synthétisées par l'intestin et le foie, les lipoprotéines contrólent, par la régulation de leurs métabolismes intravasculaire et tissulaire, la fourniture des lipides aux tissus périphériques. Ainsi, les lipoprotéines participent à la régulation de nombreuses fonctions de l'organisme : i) métabolisme énergétique des tissus ; ii) développement cellulaire (fourniture de PL et de cholestérol) ; iii) biosynthèse de médiateurs biologiques stéroïdiens (à partir du cholestérol).

Les lipoprotéines des ruminants sont caractérisées par la prédominance des lipoprotéines de haute densité (HDL, $80 \%$ ) qui exercent un rôle majeur dans le transport du cholestérol entre le foie et les tissus périphériques. Le rôle métabolique limité des lipoprotéines de faible densité (LDL, 10-15\%) chez les ruminants s'explique par le faible taux de conversion vasculaire des lipoprotéines de très basse densité (VLDL, $5-10 \%$ ) en LDL par la TG lipase hépatique et une capacité très limitée de transfert d'EC des HDL vers les LDL.

L'étude des propriétés physicochimiques des lipoprotéines bovines a révélé la présence originale de certaines particules HDL de même den- sité que les LDL. Leur composition en apoprotéines est marquée par l'abondance des apo A-I et $B$, l'absence des apo A-II et $E$ et par l'existence, inhabituelle, d'une aproprotéine de PM 51 $\mathrm{kDa}$ de propriétés comparables à celle de l'apo $\mathrm{E}$ des autres mammifères.

La nature et le taux de lipoprotéines synthétisées et sécrétées varient fortement avec les conditions hormonales et nutritionnelles. Faiblement représentées (avec les LDL) chez le footus, les HDL s'accumulent avec un maximum 15 j après la naissance, puis se stabilisent après 60 j. Leur teneur n'est ni modifiée par le sevrage ni par la maturité sexuelle des animaux, mais augmente fortement en début de lactation. La sécré. tion de VLDL augmente avec la teneur en TG etrou en cholestérol de la ration. Elle reste faible au niveau hépatique, ce qui explique le développement d'infiltration graisseuse en période de déficit énergétique (début de lactation), mais cette sécrétion peut être stimulée par la méthionine chez le veau et la vache laitière.

La captation tissulaire des LDL s'effectue via le récepteur apo $B / E$ (caractérisé chez le bovin) principalement au niveau du foie et des masses musculaires. Elle augmente fortement en période d'hyperœstrogénémie (fin de gestation) mais est réprimée chez le veau en situation d'hypercholestérolémie. L'échange de cholestérol des HDL avec les tissus s'effectuerait via un récepteur spécifique de l'apo A-I-HDL (identifié chez le bovin) mais n'impliquerait pas la captation et l'internalisation des particules HDL.

* Ce thème a fait l'objet d'une revue bibliographique parue dans J Dairy Sci (1993) 76, 3864-3881 